\title{
Label-free mapping of microstructural organisation in self-aligning cellular collagen hydrogels using image correlation spectroscopy
}

\author{
Kathleen Sanen a, Rik Paesen a, Sander Luyck a, James Phillips ${ }^{\text {b }}$, Ivo Lambrichts ${ }^{\text {c, Wendy }}$ \\ Martens ${ }^{\mathrm{c}}$, Marcel Ameloot ${ }^{\mathrm{a}}$
}

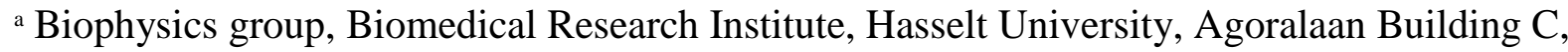
3590 Diepenbeek, Belgium

${ }^{\mathrm{b}}$ Biomaterials \& Tissue Engineering, UCL Eastman Dental Institute, University College London, 256 Gray's Inn Road, London WC1X 8LD, UK

c Morphology group, Biomedical Research Institute, Hasselt University, Agoralaan Building C, 3590 Diepenbeek, Belgium
\end{abstract}

Corresponding author: marcel.ameloot@uhasselt.be Phone: +32 112692 33. Fax: +32 11 269299.

\begin{abstract}
Hydrogels have emerged as promising biomaterials for regenerative medicine. Despite major advances, tissue engineers have faced challenges in studying the complex dynamics of cellmediated hydrogel remodelling. Second harmonic generation (SHG) microscopy has been a pivotal tool for non-invasive visualization of collagen type I hydrogels. By taking into account the typical polarization SHG effect, we recently proposed an alternative image correlation spectroscopy (ICS) model to quantify characteristics of randomly oriented collagen fibrils. However, fibril alignment is an important feature in many tissues that needs to be monitored for effective assembly of anisotropic tissue constructs. Here we extended our previous approach to include the orientation distribution of fibrils in cellular hydrogels and show the power of this model in two biologically relevant applications. Using a collagen hydrogel contraction assay, we were able to capture cell-induced hydrogel modifications at the microscopic scale and link these to changes in overall gel dimensions over time. After $24 \mathrm{~h}$, the collagen density was about
\end{abstract}


3 times higher than the initial density, which was of the same order as the decrease in hydrogel area. We also showed that the orientation parameters recovered from our automated ICS model match values obtained from manual measurements. Furthermore, regions axial to cellular processes aligned at least 1.5 times faster compared with adjacent zones. Being able to capture minor temporal and spatial changes in hydrogel density and collagen fibril orientation, we demonstrated the sensitivity of this extended ICS model to deconstruct a complex environment and support its potential for tissue engineering research.

Keywords: Collagen type I hydrogel, second harmonic generation, image correlation spectroscopy, fibril alignment, tissue engineering 


\section{Introduction}

Hydrogels are three-dimensional (3D) networks formed by natural or synthetic cross-linked polymers. Their high water-absorbing capacity and modifiable biomechanical and biochemical properties make them highly suitable carriers for different cell types [1, 2]. Therefore, many efforts have been made to use hydrogels in tissue engineering applications. By combining multidisciplinary strategies based on material, life and engineering sciences, research in this field aims to restore, preserve or enhance tissue structure and function following injury or disease [3]. Because their stiffness can range from 0.1-500 $\mathrm{kPa}$ [4], hydrogels are especially appealing for regenerating soft tissues such as skin, tendons, muscles and nerves [5].

In the past decade, natural hydrogels have gained significant interest due to their native-like extracellular matrix (ECM) properties and inherent biocompatibility $[1,2]$. Of all natural polymers, collagen type I has received great attention in tissue engineering as it is the most abundant ECM protein in the human body. Although many tissues such as corneas, vessel walls, tendons and nerves contain collagen type I, their mechanical strength and function are related to specific alignment patterns of these fibrils [6]. In the body, collagen type I is capable of selfaggregation and crosslinking to form a tissue-specific anisotropic ECM. In vitro assembly, however, consistently creates a randomly oriented fibrillar hydrogel network [7].

Different methods have been probed to generate aligned collagen hydrogels including drainage [8], microfluidic channels [9] and the use of electrical gradients [10] or magnetic fields [11]. Whereas these scaffolds are organised by being subjected to external mechanical forces, a more natural approach involves uniaxial constrained cell-seeded collagen hydrogels where cellgenerated tension causes self-alignment of both cells and collagen fibrils $[12,13]$. The use of these highly organised cellular collagen constructs for nerve repair is well studied [14-16], but little is known about the progression of these cell-induced changes in hydrogel architecture. Live imaging of the formation of aligned tissue-engineered cellular constructs will advance our 
understanding of the process and provide valuable new information to inform the construction of better 3D hydrogel microenvironments that mimic native ECM. Although many optical microscopy techniques can visualize individual cells in their ECM, most of them require exogenous dyes which could have phototoxic effects and perturb native cellular behaviour [17]. In order to truly understand ECM remodelling by embedded cells, it is essential to continuously monitor cell-matrix interactions within the 3D construct in a label-free manner.

Collagen type I fibrils are capable of generating two types of intrinsic optical signals: autofluorescence and second harmonic generation (SHG) $[18,19]$. Both processes can be induced by femto-second pulsed laser light but the resulting signals differ in wavelength and intensity. The frequency-doubled SHG signal has a much higher signal-to-noise ratio compared to the Stokes-shifted autofluorescence signals [20]. Yielding high contrast and submicron resolution images on a non-invasive basis, SHG microscopy holds great promise in the field of biomedical imaging [21] and tissue engineering [22, 23].

When studying collagen fibril organisation, extraction of quantitative information from these SHG images is not trivial. Often, time consuming manual data extraction is used which might suffer from subjective interpretation. To overcome this possible bias, image correlation spectroscopy (ICS) has been used to predict bulk mechanical properties of collagen hydrogels in an automated and objective manner. By calculating the autocorrelation function (ACF) of a fibrous SHG image, quantitative parameters such as pore size, collagen density, fibril length, thickness and orientation can be extracted [24-27]. Recently, we proposed an alternative ICS model for random fibril orientation which included SHG-specific polarization effects to obtain a more accurate ACF amplitude recovery as shown by simulations and experimental data on a collagen type I hydrogel dilution series [28].

In the current work, we expand our previous approach and describe the extension towards the characterization of cellular self-aligning collagen hydrogels designed for tissue engineering. 
The power of the extension is demonstrated in two relevant applications. First, we perform a collagen hydrogel contraction assay to evaluate cell-mediated hydrogel changes over time at the macroscopic and microscopic level. We explore the sensitivity of the extended ICS model by using two different but related cell types that can exhibit minor differences in contractile capacity, namely human dental pulp stem cells (hDPSCs) and their glial differentiated derivatives (d-hDPSCs) [15]. Secondly, we examine whether this model can be used to estimate fibril orientation in cellular hydrogels. To test the accuracy of the estimated orientation parameters, we implement a validation experiment in which manually obtained and automated (ICS) outcomes are compared. Finally, we apply the ICS model to quantify and map collagen fibril organisation in a self-aligning d-hDPSCs containing hydrogel for neural tissue engineering. 


\section{Materials and methods}

\subsection{Materials and products}

All products were purchased from Sigma-Aldrich (Bornem, Belgium) unless stated otherwise.

\subsection{Cell culture}

Human third molars were collected from donors (15-20 years of age) undergoing extraction for orthodontic or therapeutic reasons at Ziekenhuis Oost-Limburg, Genk, Belgium. The medical ethical committee of Ziekenhuis Oost-Limburg approved this study on February $3^{\text {rd }} 2014$ and written informed consent from all donors, or from legal guardians in case of under-aged donors, was obtained. hDPSCs were isolated, cultured and differentiated towards Schwann-like cells (d-hDPSCs) as described by Martens et al. [15].

\subsection{Aligned cellular hydrogels}

A tethered cell-seeded collagen gel was prepared according to methods described previously [15, 29-31] with some modifications. Briefly, gels were prepared on ice by mixing 1 volume of 10x MEM with 8 volumes of type I rat tail collagen $(2 \mathrm{mg} / \mathrm{ml}$ in $0.6 \%$ acetic acid; First Link, Wolverhampton, UK). The $\mathrm{pH}$ of the mixture was neutralized dropwise using $1 \mathrm{M}$ sodium hydroxide, after which 1 volume of d-hDPSCs suspension was added to give a final seeding density of $10^{6}$ cells $/ \mathrm{ml}$. The resulting mixture was cast within an ice-cold rectangular stainless steel mould (37 mm x $27 \mathrm{~mm} \times 4.5 \mathrm{~mm}$ ) and tethered at each end through the use of a porous mesh. The gels were allowed to set for $15 \mathrm{~min}$ at $37^{\circ} \mathrm{C}$, transferred to a $\# 1.5$ glass bottom petri dish and subsequently immersed with standard culture medium. From this moment on $(\mathrm{t}=0 \mathrm{~h}$ after casting), tethered gels with initial dimensions of $16 \mathrm{~mm}$ x $6.5 \mathrm{~mm}$ x $4.5 \mathrm{~mm}$ were kept at $37{ }^{\circ} \mathrm{C}$ in a humidified atmosphere containing $5 \% \mathrm{CO}_{2}$ in a cell culture incubator or in the microscope stage incubator for imaging at $0,4,8$ and $32 \mathrm{~h}$ after casting. The orientation of the $3 \mathrm{D}$ construct is referred to as $\mathrm{x}$ for the long axis of gel, $\mathrm{y}$ for the shorter axis and $\mathrm{z}$ for the height. 
The mould was always positioned such that the $\mathrm{x}$-axis of the hydrogel was parallel to the polarization of the incident light, which is always along the x-axis of the image in this paper. Since alignment occurs in the direction of tension generated by the cellular gel contraction being resisted by the tethering bars, elongated cells and aligned collagen fibrils along the $\mathrm{x}$-axis are expected in the acquired images. Per hydrogel, 6 cells were randomly selected in the central part of the tethered system and around each cell, 3 defined regions near the cellular processes (axial, diagonal and parallel with respect to cellular processes) (Fig. 3a) were imaged. Differentiated hDPSCs from 4 different donors were used to carry out 4 independent experiments $(n=4)$.

\subsection{Hydrogel contraction assay}

Gels were prepared as described above with some modifications. Donor-matched hDPSCs or d-hDPSCs were added to the hydrogel mixture in a final seeding density of $10^{6}$ cells $/ \mathrm{ml}$. Cellseeded collagen hydrogels were cast in duplicate in a 96-well plate (Greiner F-bottom; 75 $\mu \mathrm{l} /$ well) for six different time points $(0,2,4,6,8$ and 24 hours $)$. After $10 \mathrm{~min}$ of setting at 37 ${ }^{\circ} \mathrm{C}$, gels were immersed in $200 \mu \mathrm{l}$ standard culture medium and detached from the wells using a fine spatula. Four independent experiments, i.e. cells from four different donors $(n=4)$, were carried out and for each experiment, free-floating hydrogel contraction was assessed both macro- and microscopically at the indicated time points. For macroscopic analysis, the medium was removed and digital images were taken. ImageJ software was used to determine the area of the upper surface of the gels. Collagen gel size was defined as a percentage of the initial hydrogel area. For microscopic analysis, duplicate gels were transferred to $\mu$-Slide 8 Well ibiTreat (Proxylab, Beloeil, Belgium) and 3 random regions without cells in the field of view were imaged in the centre of each hydrogel. For one donor $(n=1)$, also 3 random regions at the edge of each hydrogel were imaged. 


\subsection{Microscopy}

Label-free imaging of cell-seeded gels was performed using a Zeiss LSM510 META (Carl Zeiss, Jena, Germany) mounted on an Axiovert 200M. The horizontally polarized excitation was provided by a femtosecond pulsed laser (MaiTai DeepSee, Spectra- Physics, CA, USA) tuned to a central wavelength of $810 \mathrm{~nm}$. The beam was reflected by a short-pass $650 \mathrm{~nm}$ dichroic beam splitter and focused onto the sample with a 40x/1.1 water immersion objective (LD C-Apochromat 40x/1.1W Korr UV-VIS-IR, Carl Zeiss). The average excitation power at the sample is approximately $4 \mathrm{~mW}$. Two-photon excitation (TPE) autofluorescence of the embedded cells and SHG signals from the collagen fibrils were epi-collected, discriminated with a $442 \mathrm{~nm}$ dichroic beam splitter and transmitted through a $100 \mathrm{~nm}$ or $5 \mathrm{~nm}$ wide band pass filter with a central wavelength of $550 \mathrm{~nm}$ or $405 \mathrm{~nm}$ respectively. An analogue photomultiplier tube (Zeiss) was used for detection in non-descanned mode. Based on our previous work [28], a pixel size of $85 \mathrm{~nm}$ was chosen for 1024 x 1024 pixels per image, yielding a field of view of $87 \mu \mathrm{m} \times 87 \mu \mathrm{m}$ which proved to be sufficient to correctly estimate all ACF parameters and provides sufficiently detailed spatial insight in the studied structures. If we would reduce the field of view (FOV), while keeping the pixel size constant, less fibrils are included in the image. Because ICS is a statistical method, lowering the number of fibrils would result in less accurate ACFs, and therefore less representative parameter values would be recovered from the ACF. Conversely, increasing the FOV with fixed pixel size would yield better defined ACFs, but also averages more local spatial variations. The images were taken $20 \mu \mathrm{m}$ above the cover glass and microscopy was performed at $37^{\circ} \mathrm{C}$.

\subsection{ACF analysis}

The analysis of the ACF is based on the previously described ICS model [28] developed to study hydrogels imaged by SHG. In the extended model used in the current work, the ACF $\mathrm{g}(\eta, \xi)$ is modeled by 


$$
\mathrm{g}(\eta, \xi)=\mathrm{g}_{00}\left|\sum_{i=1}^{N} \mathrm{~g}_{\theta_{i}}^{1}(\eta, \xi, L) m\left(\theta_{i}\right)\right|
$$

with $\mathrm{g}_{\theta_{i}}^{1}(\eta, \xi, L)$ the ACF of a single fibril of length $L$ oriented at an angle $\theta_{i}$ relative to the orientation of the polarization direction of the incident light. The angles $\theta_{i}$ are set to be equally spaced at $10^{\circ}$ intervals between $0^{\circ}$ and $180^{\circ}$. The notation $|\ldots|$ takes care of the normalization of the sum to unity such that the ACF amplitude is solely defined by $g_{00}$. The amplitude $g_{00}$ is inversely proportional to the fibril density [28].

The modulation function $m(\theta)$ in eq. (1) accounts for the polarization effect related to SHG imaging and the orientation distribution of the fibrils,

$$
m(\theta)=p^{2}(\theta) \Phi(\theta)
$$

with

$$
p(\theta) \propto \sin 2 \theta+\left(\frac{d_{31}}{d_{15}} \sin ^{2} \theta+\frac{d_{33}}{d_{15}} \cos ^{2} \theta\right)^{2}
$$

where $d_{i j}$ are non-zero second order macroscopic susceptibility tensor elements of the collagen fibrils and where $\Phi(\theta)$ describes the orientation distribution of the fibrils within the image. We assume that the fibril orientations have a Gaussian distribution around the preferential angle $\mu$. To implement the Gaussian distribution as a wrapped distribution function, a circular variant analogue to the so called Von Mises distribution was used. Since the fibrils have no sense, a periodicity of $\pi$ appears in the ACF. Therefore, the standard Von Mises distribution with a periodicity of $2 \pi$ must be adjusted to one with half the periodicity. Our implementation of the adjusted Von Mises distribution reads

$$
\Phi(\theta) \propto \frac{e^{-\kappa \cos 2(\theta-\mu)}}{I_{0}(\kappa)}
$$

with $I_{0}(\kappa)$ the zeroth order Bessel function of the first kind, and $\kappa$ a dimensionless parameter representing the spread of the distribution, being analogue to the inverse of four times the variance of the approximated wrapped Gaussian distribution. This means that for $\kappa=0$, a 
uniform distribution is obtained, while for increasing values of $\kappa$ a Gaussian distribution with a standard deviation of $0.5 \kappa^{-0.5}$ is approximated. Note that for both $p(\theta)$ and $\Phi(\theta)$ only the proportionalities are used, since additional factors vanish by the ACF normalization.

In the actual analysis of the experimental ACF, the tensor element ratios are fixed to the values we determined previously.: $d_{31}=1.5 d_{15}$ and $d_{33}=1.8 d_{15}$ [28]. The $e^{-2}$ width of the point spread function was fixed at $0.33 \mu \mathrm{m}$. The freely adjustable parameters in the fitting procedure are the ACF amplitude $g_{00}$, the average fibril length $L$, the preferential orientation $\mu$ and the orientation spread $\kappa$. Consistently with our previous work [28], the length always results in values above the detection limit. Therefore, we cannot draw conclusions on the fibril lengths. To account for possible background effects, an additional offset is included in the fitting procedure as well. The fitting is done on the central 64x64 pixels of the ACF. The analysis was done with in house developed Matlab (The MathWorks) routines. The analysis of one ACF takes approximately 10 seconds on a standard pc with a quad-core processor (Intel i5) running at $3.30 \mathrm{GHz}$.

\subsection{Validation of ICS analysis for orientation parameters $\kappa$ and $\mu$}

Three random images $(n=3)$ were collected from the central part of a tethered gel containing $\mathrm{d}$-hDPSCs at each of the indicated time points (see section 2.3). To minimize heterogeneity within a SHG image, pictures were captured without cells or their processes in the field of view. For validation purposes, collagen fibrils were traced manually in ImageJ software. For each image, the distribution of fibril orientations was analyzed using the Von Mises distribution given by eq. (4), yielding the mean direction $\mu$ and the concentration parameter $\kappa$. These parameters were then compared to their corresponding parameters returned by the automated ICS analysis described in section 2.6. 


\subsection{Statistical analysis}

Statistical analysis was performed using Graphpad Prism 5 software (Graphpad, California, USA). Data from the hydrogel contraction assay and the aligned hydrogel constructs were compared by means of a two-way ANOVA followed by Bonferroni's multiple comparison test. $\mathrm{P}$-values $\leq 0.05$ were considered statistically significant $(* \mathrm{p} \leq 0.05 ; * * \mathrm{p} \leq 0.01 ; * * * \mathrm{p} \leq$ 0.001). All data were expressed as mean \pm Standard Error of the Mean (SEM). 


\section{Results}

\subsection{Differential hydrogel contraction on macro and micro scale}

A hydrogel contraction assay was performed to determine the sensitivity of the ICS model in a biologically relevant setting. Since the macroscopic change in overall gel dimensions is a result of cell-level activity [32], it should be possible to link macroscopic observations to microscopic data. Therefore, contraction of collagen type I hydrogels was captured macro- and microscopically for both hDPSCs and d-hDPSCs at six different time points. For macroscopic evaluation, hydrogel contraction was measured from digital pictures (

a; upper row) as a percentage of the initial hydrogel area (

b). While contraction after $2 \mathrm{~h}$ was similar for both cell types ( $~ 80 \%$ of initial area), hydrogels seeded with d-hDPSCs were significantly smaller compared to hydrogels seeded with hDPSCs at $4 \mathrm{~h}(64 \%$ versus $76 \%$ of initial area) and $6 \mathrm{~h}$ after casting (and 52\% vs $65 \%$ of initial area). After $24 \mathrm{~h}$, a reduction to $\sim 40 \%$ of the original hydrogel size was observed for both cell types.

To evaluate whether this differential macroscopic hydrogel contraction by hDPSCs and dhDPSCs corresponds to distinct changes in matrix density, SHG imaging at the centre of these gels (

a; lower row) with subsequent ICS analysis was performed.

c shows the ACF amplitude $g_{00}$ as function of time. Starting off with the same collagen density directly after casting $(\mathrm{t}=0 \mathrm{~h})$, a remarkable difference was observed after $2 \mathrm{~h}$ where hydrogels seeded with d-hDPSCs showed a significantly lower amplitude compared to gels seeded with hDPSCs. $4 \mathrm{~h}$ after hydrogel casting the amplitude was comparable for both conditions but another $2 \mathrm{~h}$ later, d-hDPSCs-containing gels had a significantly higher collagen density 
compared to gels with hDPSCs as indicated by a lower amplitude ( $\mathrm{g}_{00}=0.31$ and 0.65 respectively) (

c). In the end, both cellular hydrogels reached a $\mathrm{g}_{00}$ value of $\sim 0.30$. It must be noted that at the edge of the hydrogel, this minimal ACF amplitude is already reached $2 \mathrm{~h}$ after hydrogel casting with hDPSCs and d-hDPSCs (Supplementary figure). Although both cell types showed similar patterns for hydrogel contraction (

c, full line), hDPSCs showed a time lag in initiation of central collagen fibril condensation (

c, dotted line). Taken together, the differential decrease in area between hydrogels seeded with hDPSCs or d-hDPSCs is coupled with proportional increases in collagen density as indicated by the trend of distinctively decreasing $\mathrm{g}_{00}$ values.

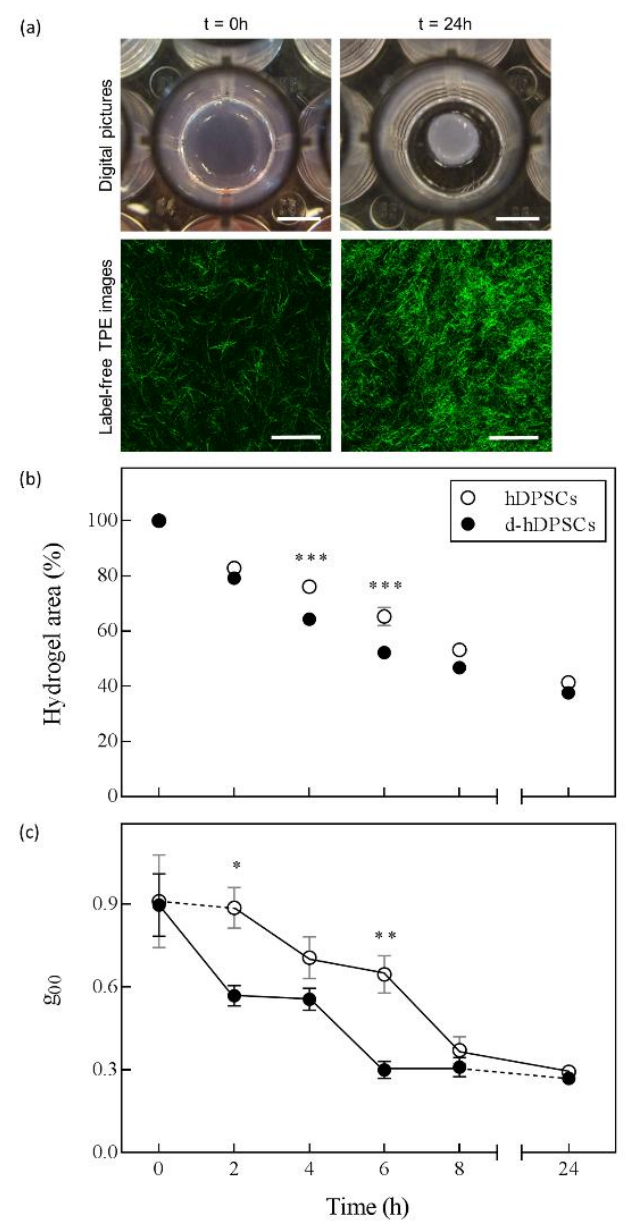


Fig. 1: Hydrogel contraction assay with hDPSCs or d-hDPSCs. Each free-floating collagen type I hydrogel seeded with (d-)hDPSCs was monitored from directly after hydrogel casting $(\mathrm{t}=0 \mathrm{~h})$ to the fully contracted stage $(\mathrm{t}=24 \mathrm{~h})$. (a) Digital pictures (scale bars $=3 \mathrm{~mm}$ ) show the macroscopic changes in hydrogel area while labelfree TPE images (scale bars $=20 \mu \mathrm{m}$ ) visualize collagen type I fibrils (green) by SHG signals. (b) Macroscopic evaluation was done by measuring the area of the hydrogel from digital images, depicted as percentage of the initial hydrogel area as a function of time. (c) Microscopically obtained SHG images with a pixel size of $85 \mathrm{~nm}$ and 1024 x 1024 pixels per image (yielding a field of view of $87 \mu \mathrm{m}$ x $87 \mu \mathrm{m}$ ) were analyzed by the ICS model described in section 2.6, of which the ACF amplitude $\mathrm{g}_{00}$ is plotted as a function of time. hDPSCs and d-hDPSCs showed similar changes in $\mathrm{g}_{00}$ values (full lines) with a small time lag (dotted lines) between both cell types. Data represent means $\pm \operatorname{SEM}(\mathrm{n}=4) * \mathrm{p}<0.05, * * \mathrm{p}<0.01, * * * \mathrm{p}<0.001$ compared with hDPSCs at each time point. The error bars are often smaller than the symbol.

\subsection{Validation of orientation parameters}

For the characterization of fibrillar hydrogels, not only collagen density but also fibril orientation are of interest, especially when developing aligned tissue engineered constructs. We recently showed that d-hDPSCs were able to self-align in a tethered collagen type I hydrogel [15], but the effect on local hydrogel architecture remained to be elucidated. Here, these constructs were monitored over time by TPE autofluorescence of the embedded cells and SHG microscopy of the collagen fibrils. Directly after casting $(t=0 \mathrm{~h})$, cells appeared round and collagen fibrils seemed to be randomly oriented (Fig. 2a; left). However, when the hydrogel is completely contracted $(t=32 \mathrm{~h})$, alignment of both cells and collagen fibrils was observed parallel to the longitudinal axis of the 3D construct (Fig. 2a; right).

As this is the first time that the Von Mises distribution has been included in an ICS based approach, we first determined the validity of the method to quantify the degree of fibril alignment. Using a set of SHG images from an aligning hydrogel, orientation parameters obtained by automated analysis and manual tracing were compared. Representative manually 
determined fibril orientation histograms with fitted Von Mises function at $0 \mathrm{~h}$ and $32 \mathrm{~h}$ after hydrogel casting are shown in Figure 2b. Both analysis methods yielded essentially equal values in terms of the preferential angle $\mu$ and the spread of angle distribution $\kappa$ (Fig. 2c-d). Being inversely proportional to four times the variance of the approximated wrapped Gaussian distribution, a $\kappa$ value near zero is indicative of a large spread of angle distribution for the collagen type I fibrils while increasing $\kappa$ values point to a narrow distribution around a preferential direction of fibril orientation. The increasing value of $\kappa$ over time (Fig. 2c) indicates that the organisation of collagen type I fibrils in the tethered hydrogel changes from random directly after gel formation to highly aligned after contraction. As at $\mathrm{t}=0 \mathrm{~h}$ the fibrils are expected to be in random orientation [28], the average value of $\mu$ is about zero with a substantial spread over the separate determinations (Fig. 2d). The time evolution of $\kappa$ and $\mu$ indicate that the collagen fibrils orient along the $\mathrm{x}$-axis of the mould with a distribution with decreasing width under the action of the embedded cells. 
(a)

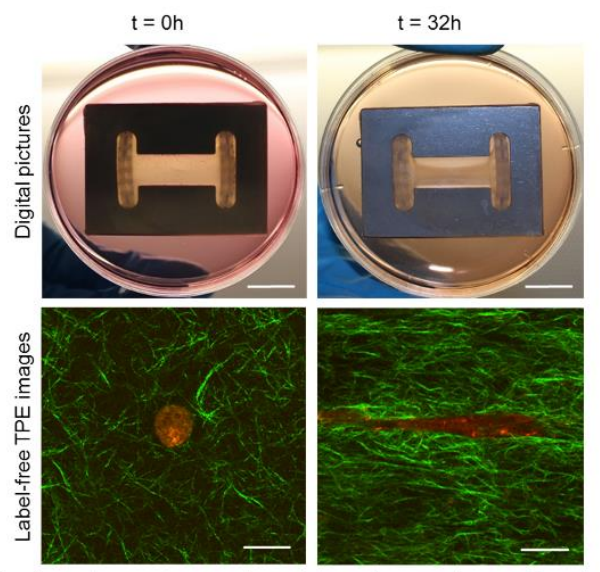

(b)

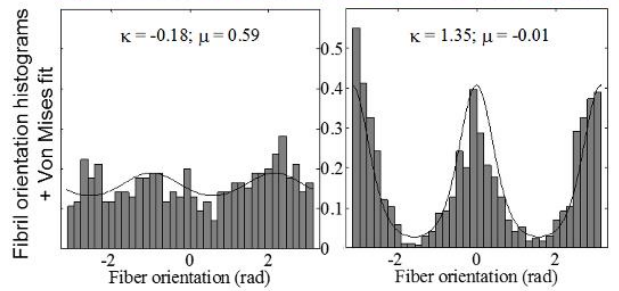

(c)

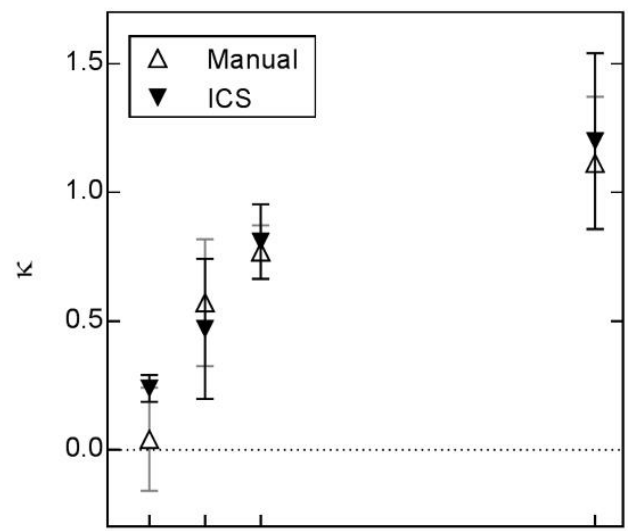

(d)

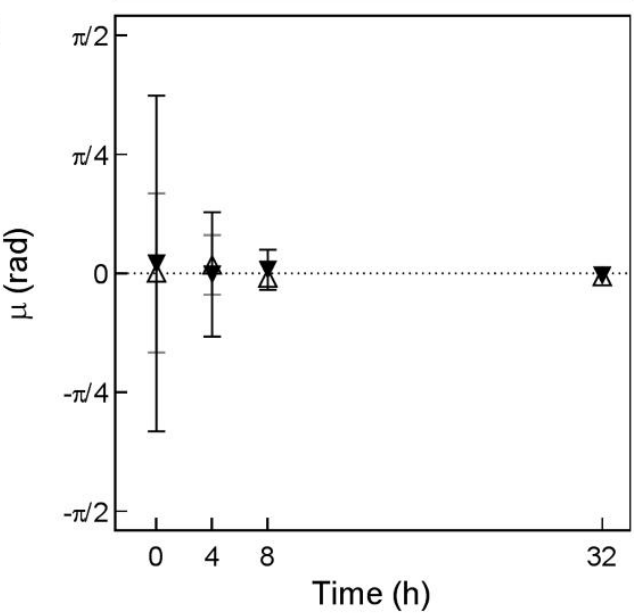

Fig. 2: Validation of ICS analysis of SHG images to quantify fibril orientation. (a) Each tethered collagen type I hydrogel seeded with d-hDPSCs was monitored from directly after hydrogel casting $(\mathrm{t}=0 \mathrm{~h})$ to the fully contracted stage $(t=32 \mathrm{~h})$. Digital pictures (scale bars $=10 \mathrm{~mm}$ ) show the macroscopic changes in hydrogel area while label-free TPE images (scale bars $=20 \mu \mathrm{m}$ ) visualize the cells (red) and collagen type I fibrils (green) by 
TPE autofluorescence and SHG signals respectively. (b) Representative manually determined fibril orientation histograms with fitted Von Mises function at $0 \mathrm{~h}$ and $32 \mathrm{~h}$ after hydrogel casting. At 4 different time points, (c) the spread of fibril orientation $\kappa$ and (d) the preferential angle $\mu$ were calculated by manual and ICS analysis of SHG images with a pixel size of $85 \mathrm{~nm}$ and 1024 x 1024 pixels per image (yielding a field of view of $87 \mu \mathrm{m} \times 87 \mu \mathrm{m}$ ). Data represent means $\pm \operatorname{SEM}(n=3)$. The error bars are sometimes smaller than the symbol.

\subsection{Local changes in hydrogel architecture during cell-mediated alignment}

As described above, an overall time-dependent increase in fibril organisation was observed for tethered hydrogels (Fig. 2). However, since this collagen remodeling is cell-mediated, local differences in hydrogel architecture can be expected. To explore this hypothesis, three different zones near cells were imaged: axial, diagonal and parallel with respect to the leading edge (Fig. 3a; red, black and blue square respectively). Fig. $3 \mathrm{~b}$ shows an exponential fit with a characteristic relaxation time $\tau$ for the ACF amplitude $\mathrm{g}_{00}$ as a function of time. For each of the above mentioned zones, the ACF amplitude decreases over time, indicating an increase in collagen density. A characteristic time of $\sim 3.4 \mathrm{~h}$ is obtained for all zones, reaching the maximum collagen density $8 \mathrm{~h}$ after hydrogel casting (Fig. 3b).

The spread of the fibril orientation decreases over time as indicated by an increasing $\kappa$ (Fig. 3c). Directly after casting $(t=0 \mathrm{~h})$, the low $\kappa$ values of $\sim 0.4$ for the three zones reflect an isotropic environment, thereby making the mean fibril direction $\mu$ at this time point meaningless (Fig. 3c-d). As time progresses, zones axial to the leading edge have significantly higher $\kappa$ values compared to diagonal $(\mathrm{t}=4-8 \mathrm{~h})$ and parallel $(\mathrm{t}=4-8-32 \mathrm{~h})$ zones (Fig. $3 \mathrm{c})$. In addition, the characteristic times of $\kappa$ reveal that axial hydrogel zones tend to align $\sim 1.5$ times and $\sim 1.8$ times faster compared to diagonal and parallel zones respectively. In this situation, Fig. $3 \mathrm{~d}$ demonstrates that the preferential direction of fibril orientation is along the $\mathrm{x}$-axis of the construct $(0 \mathrm{rad})$ for all zones considered. 
(a)
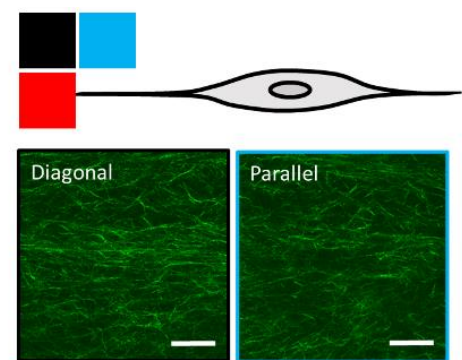

Parallel

Axial

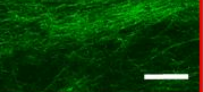

(b)

(c)
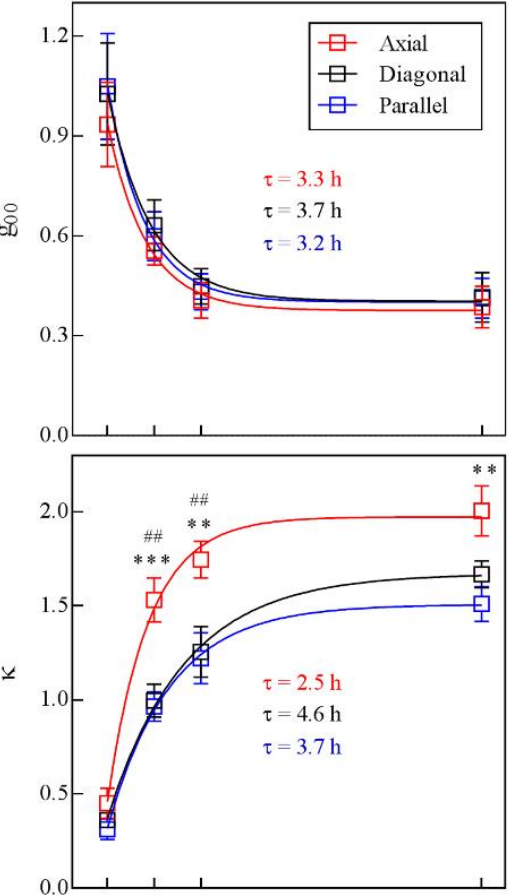

(d)

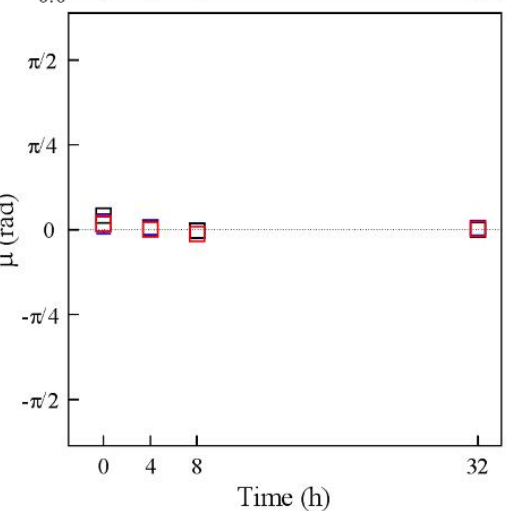

Fig. 3: Detection of local changes in cell-mediated hydrogel alignment by ICS analysis. (a) Schematic representation of the different zones near cellular processes in a tethered hydrogel, further referred to as axial (red), diagonal (black) and parallel (blue) zones. Per zone, a representative SHG image at $4 \mathrm{~h}$ after hydrogel casting is depicted. Panels (b-d) respectively show the ACF amplitude $\mathrm{g}_{00}$, spread on fibril orientation $\kappa$ and preferential 
angle $\mu$ over time for each of the zones obtained by ICS analysis of SHG images with a pixel size of $85 \mathrm{~nm}$ and 1024 x 1024 pixels per image (yielding a field of view of $87 \mu \mathrm{m}$ x $87 \mu \mathrm{m})$. Data represent means \pm SEM $(n=4)$. $* *$ and ${ }^{\# \#} \mathrm{p}<0.01, * * *$ and ${ }^{\# \#} \mathrm{p}<0.001$ for the axial zone compared to the diagonal $(\#)$ or parallel $(*)$ zone at that time point. The error bars for panel (d) are smaller than the symbol. 


\section{Discussion}

The success of tissue engineering is often dependent on the similarity of the 3D construct and the native ECM. So, in order to develop biologically active scaffolds for regenerative medicine purposes, their microstructural organisation must be thoroughly studied during the design process and before implantation, preferentially by using non-invasive imaging and robust automated analysis methods. In this study, we extended our previously developed ICS-based model [28] to get the orientation distribution of fibrils within images obtained by SHG microscopy. The power of this model is shown in two relevant applications where we quantify the spatial and structural characteristics of collagen type I fibrils within different cellular hydrogel systems over time.

Cell-mediated contraction of free-floating collagen type I hydrogels has been well described in literature. Being dependent on the collagen concentration, the number of cells and the cell type, in vitro collagen contraction assays are amongst others extensively performed in tissue engineering research to optimize the composition of newly developed 3D constructs [33, 34]. Although hydrogel size and opacity are currently the most conventional readout parameters of this assay, relevant microstructural information cannot be captured in this way. The current work shows that ICS analysis of SHG images can overcome this limitation with high accuracy and without interfering in the contraction process as the method is optical and label-free. As expected, hydrogel contraction by hDPSCs or d-hDPSCs resulted in a decrease in hydrogel area. This surface reduction was accompanied by a measurable increase in collagen density as shown by declining $\mathrm{g}_{00}$ values. It must be noted, however, that during the first 2 hours of hydrogel contraction by hDPSCs, the central collagen density remained unchanged despite a reduction in hydrogel area. Since hydrogel compaction has been described to propagate from the edges of the hydrogel into the bulk [35], we investigated whether contraction of our freefloating hydrogels also occurred in a non-uniform manner. Indeed, when measuring the ACF 
amplitude in the centre and the edge of hydrogels at different time points, collagen condensation at the edge of the hydrogel started immediately after casting and reached its maximal density after 2 hours. This boundary effect explains why the initial area of hDPSC-seeded hydrogels decreases without a reduction in the central collagen density. Another observation was that both cell types showed stepwise changes in collagen density and a gradual reduction in hydrogel area. At certain time points, however, significant smaller hydrogel areas and $g_{00}$ values were detected for hydrogels seeded with d-hDPSCs compared to hDPSCs. So despite the similar patterns for hydrogel contraction, d-hDPSCs were more efficient in contracting collagen type I hydrogels. It must be noted that the collagen density was significantly higher for d-hDPSCs already after 2 hours, while a significant difference in hydrogel area was only observed after 4 hours, pointing to a time lag between locally initiated hydrogel remodeling and the macroscopic effect. In the course of time, both cell types reach a final collagen density that is about three times higher than the initial density, which is of the same order as the decrease in hydrogel area observed macroscopically.

In addition to change in fibril density, cell and matrix alignment is an important feature that needs to be monitored and understood for effective construction of anisotropic tissue constructs. The two-dimensional discrete Fourier transform has been shown to be effective in quantifying the degree of collagen fibril organisation in different biological tissues [36-38]. In this work, we present an alternative by extending our previously described ICS model [28], that provided a measure for the collagen fibril density, with the Von Mises distribution yielding the nonphenomenological parameter $\kappa$ which represents the spread of fibril orientation. By comparison with manually obtained data, we showed the validity of this adapted ICS model for the determination of the orientation parameters $\mu$ and $\kappa$. Both the Fourier based approach and the ICS approach hold the same constraints regarding the size of the analyzed FOV, which was already discussed before for the ICS case. Yet, the preferred FOV size also depends on whether 
local or more global information is desired. Similarly to the Fourier based approach [38], the ICS technique can also be used to map local fibril orientation by subdividing the full FOV into smaller regions of interest (ROIs). For this type of application, the main orientation $\mu$ returned by the ICS approach is more valuable than the orientation spread $\kappa$ for each ROI. When interested in the non-random fibril orientation distribution, also both the Fourier and the ICS approach can be applied. In the Fourier approach, increasing anisotropy yields more elongated frequency spectra. The degree of anisotropy can then be quantified by measuring this elongation, for instance through fitting with an ellipse and comparing the major and minor axis lengths [38]. For the discussed ICS based approach, it was shown that the orientation spread is well quantified by $\kappa$ which is related to the standard deviation of a Gaussian distribution of possible fibril orientations. The Fourier and ICS method can thus be used to both map fibril orientation and quantify the corresponding orientation distribution function.

Compared to the Fourier approach in which the spectrum is analyzed by fitting it with an ellipse [38], the ICS approach is typically slower since it is more computationally intensive in the ACF analysis step. Yet, the ICS model was specifically designed to analyze images containing fibrils, and the resulting fit parameters are directly related to physical quantities of those fibrils. Also, it was previously shown that all fibrils are taken into account when considering the ACF [28], rendering the ICS method an appropriate statistical tool to quantify the global fibril organisation, which was the goal of this work. Additionally, the model includes the SHG related polarization effect which is typically present when imaging with linearly polarized light. This phenomenon might induce artifacts in the frequency spectrum which are not accounted for in Fourier based approaches. Finally, it must be noted that the proposed ICS model is not limited to label-free SHG images only. A similar approach can also be applied to fluorescent images in case of fluorescence sensitivity to polarization, or when omitting the polarization effect in the ICS model also regular fluorescence images can be analyzed. 
Throughout the central part of the tethered hydrogels, an overall time-dependent increase in fibril organisation along their longitudinal axis was observed. However, cell-induced ECM remodeling and compaction have been shown to give rise to local heterogeneities $[35,39,40]$. To capture such non-uniformities within the collagenous matrix, we specified three arbitrary zones near cellular processes that could be differentially affected in terms of hydrogel remodeling. As these predefined zones are in the direct vicinity of the cell membrane, the first anchoring events could already induce changes in hydrogel architecture, thereby explaining the slightly higher offset values of $\kappa$ in these zones as compared to those of random locations in the validation experiment. According to our expectations, we observed that the rate at which the spread of fibril orientation decreased was higher in the axial zones compared to diagonal and parallel zones. In other words; the fibrils that are positioned directly in front of the leading edge of the cell exhibited faster alignment than those in adjacent areas. Also note that for the diagonal and parallel zones, the characteristic times of $\kappa$ were larger than those of $\mathrm{g}_{00}$, indicating that hydrogel remodeling for these regions was still ongoing while the maximum density had already been reached. Quantitative maps of collagen density, fibril orientation and the degree of alignment around individual cells are required to capture compositional heterogeneities of collagen architecture. Since not only single cells but also intercellular forces play a role in matrix remodeling [39], such contour maps can provide insight into the formation of aligned cellular collagen type I hydrogels and can be used to optimize cellular density of tissue engineered constructs.

In the present study, we have characterized 3D cell-laden hydrogel scaffolds using an ICS model based on SHG images. Being able to capture minor temporal and spatial changes in hydrogel density and collagen fibril orientation in biologically relevant systems, we showed the sensitivity of this technique to deconstruct a complex environment. Looking beyond bulk hydrogel composition is key in understanding the mechanisms that influence the mechanical 
and biological properties of artificial tissues. Therefore, we believe that this ICS method has high-throughput potential in screening arrays of hydrogel scaffolds, making it an interesting tool for future tissue engineering research.

\section{Acknowledgements}

The authors are grateful to Dr. Constantinus Politis and Dr. Luc Vrielinck (Ziekenhuis Oost Limburg, Genk, Belgium) for providing dental pulp tissue. K.S. acknowledges funding from Research Foundation Flanders [Fonds Wetenschappelijk Onderzoek (FWO), grant 11N0914N] and funding by the Interreg EMR IV-A consortium BioMIMedics (www.biomimedics.org) which is co-financed by the European Union, local governments, research institutes and SMEs. W.M. benefits from a grant via FWO (GO29112FWO) and M.A. acknowledges the Federal Science Policy of Belgium (IAP-7/05), the support by the FWO-onderzoeksgemeenschap "Scanning and Wide Field Microscopy of (Bio)-organic Systems", and the Province of Limburg (Belgium) for the financial support within the tUL IMPULS FASE II program, allowing for the upgrading of the laser source used in this work. 


\section{Bibliography}

1. Aurand ER, Lampe KJ, et al. Neuroscience research. 2011.

2. Geckil H, Xu F, et al. Nanomedicine (Lond). 2010;5(3):469-84.

3. Langer R, Vacanti JP. Science. 1993;260(5110):920-6.

4. Murphy WL, McDevitt TC, et al. Nat Mater. 2014;13(6):547-57.

5. Levental I, Georges PC, et al. Soft Matter. 2007;3(3):299-306.

6. $\quad$ Fratzl P, Weinkamer R. Prog Mater Sci. 2007;52(8):1263-334.

7. Van Der Rest M, Garrone R, et al. Collagen A family of proteins with many facets. In: Bittar E, Kleinman $\mathrm{H}$, editors. Advances In Molecular And Cell Biology; Extracellular Matrix. 6: Elsevier; 1993. p. 1-67.

8. $\quad$ Elsdale T, Bard J. J Cell Biol. 1972;54(3):626-\&

9. Lee $\mathrm{P}$, Lin R, et al. Biomed Microdevices. 2006;8(1):35-41.

10. Cheng XG, Gurkan UA, et al. Biomaterials. 2008;29(22):3278-88.

11. Torbet J, Ronziere MC. Biochem J. 1984;219(3):1057-9.

12. Eastwood M, Mudera VC, et al. Cell motility and the cytoskeleton. 1998;40(1):13-21.

13. Mudera VC, Pleass R, et al. Cell motility and the cytoskeleton. 2000;45(1):1-9.

14. Georgiou M, Bunting SCJ, et al. Biomaterials. 2013;34(30):7335-43.

15. Martens W, Sanen K, et al. FASEB J. 2014;28(4):1634-43.

16. Georgiou M, Golding JP, et al. Biomaterials. 2015;37:242-51.

17. Teraski M, Dailey ME. Confocal microscopy of living cells. Handbook of Biological Confocal Microscopy. 2 ed. New York: Plenum Press; 1995.

18. Freund I, Deutsch M, et al. Biophysical journal. 1986;50(4):693-712.

19. RichardsKortum R, SevickMuraca E. Annu Rev Phys Chem. 1996;47:555-606.

20. Brown E, McKee T, et al. Nature medicine. 2003;9(6):796-800.

21. Campagnola P. Analytical chemistry. 2011;83(9):3224-31.

22. Matcher SJ. Applications of SHG microscopy in tissue engineering. Optical Techniques in Regenerative Medicine. 1: CRC Press; 2013. p. 82-8.

23. Vielreicher M, Schurmann S, et al. J Roy Soc Interface. 2013;10(86).

24. Eichhorn SJ, Sampson WW. J Roy Soc Interface. 2005;2(4):309-18.

25. Raub CB, Unruh J, et al. Biophysical journal. 2008;94(6):2361-73.

26. Mir SM, Baggett B, et al. Biomed Opt Express. 2012;3(2):215-24.

27. Robertson C, George SC. Journal of biomedical optics. 2012;17(8).

28. Paesen R, Sanen K, et al. Acta biomaterialia. 2014;10(5):2036-42.

29. Phillips JB, Brown R. Methods Mol Biol. 2011;695:183-96.

30. Brown RA, Wiseman M, et al. Adv Funct Mater. 2005;15(11):1762-70.

31. Phillips JB, Brown RA. International Patent WO2004087231. 2004.

32. Grinnell F, Petroll WM. Annual review of cell and developmental biology. 2010;26:335-61.

33. Brown RA. Exp Cell Res. 2013;319(16):2460-9.

34. Ahearne M. Interface Focus. 2014;4(2).

35. Fernandez $\mathrm{P}$, Bausch AR. Integrative biology : quantitative biosciences from nano to macro. 2009;1(3):252-9.

36. Matteini P, Ratto F, et al. Optics express. 2009;17(6):4868-78.

37. Rao RA, Mehta MR, et al. Optics express. 2009;17(17):14534-42.

38. Ghazaryan A, Tsai HF, et al. Journal of biomedical optics. 2013;18(3):31105.

39. Gjorevski N, Nelson CM. Biophysical journal. 2012;103(1):152-62.

40. Winer JP, Oake S, et al. PloS one. 2009;4(7):e6382. 
(a)
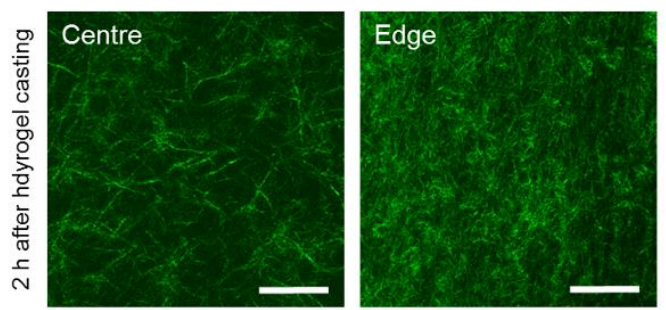

(b)

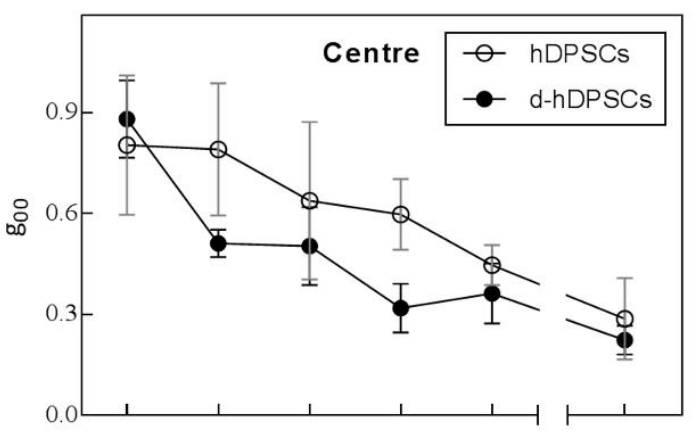

(c)

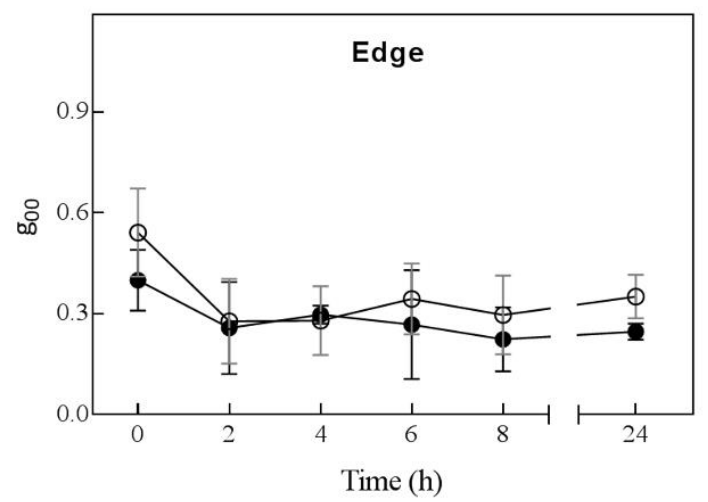

Fig. S4: Centre versus edge microstructural changes during hydrogel contraction. Each free-floating collagen type I hydrogel seeded with (d-)hDPSCs was monitored from directly after hydrogel casting $(\mathrm{t}=0 \mathrm{~h})$ to the fully contracted stage $(t=24 \mathrm{~h})$ at the centre and the edge of the hydrogel. (a) Representative SHG images of the centre and the edge of contracting hydrogels $2 \mathrm{~h}$ after casting. ICS analysis of images with a pixel size of $85 \mathrm{~nm}$ and 1024 x 1024 pixels per image (yielding a field of view of $87 \mu \mathrm{m} \times 87 \mu \mathrm{m}$ ). For both hDPSCs and d-hDPSCs, the ACF amplitude $g_{00}$ is plotted as a function of time at the centre (b) and edge (c) of the hydrogel. Data represent means \pm standard deviation ( $n=1$, i.e. only one donor used for cellular hydrogels, but duplicate hydrogels with 3 images per hydrogel analyzed). 